\title{
FEM modeling of structure and properties of diamond-SiC-(Al) composites developed for thermal management applications
}

\author{
P. Unifantowicz ${ }^{1,2, a}, T^{2}$ Boguszewski ${ }^{1, b}$, Ł. Ciupiński, c,$E$. Fortuna ${ }^{1, d}$, \\ M. Lewandowska, e, S. Vaucher ${ }^{2, f}$ and K. J. Kurzydłowski ${ }^{1, g}$ \\ ${ }^{1}$ Warsaw University of Technology, Faulty of Materials Science and Engineering, UI. Wołoska 141, \\ 02-507 Warsaw, Poland \\ ${ }^{2}$ EMPA - Swiss Federal Laboratories for Materials Testing and Research, Nanocomposites \\ Processing Group, Feuerwerkerstrasse 39, 3604 Thun, Switzerland

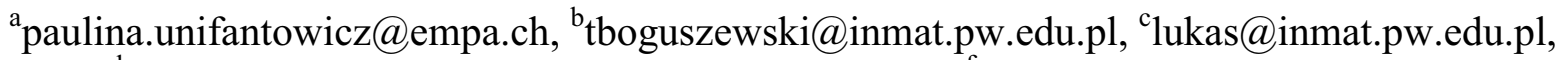 \\ delaf@inmat.pw.edu.pl, ${ }^{\mathrm{d}}$ malew@inmat.pw.edu.pl, ${ }^{\mathrm{f}}$ sebastien.vaucher@empa.ch, \\ gkjk@inmat.pw.edu.pl
}

Keywords: FEM modelling, thermal conductivity, diamond-SiC composites

\begin{abstract}
Thermal management materials frequently have multi-phase composite character with complex architecture of the constituents. As a result, design rules are needed which can be used in selection of the phases and optimizing their volume fractions. The study shows that such are provided by finite element modeling of these composites. This is demonstrated for a diamond-SiC-Si-(Al) composites, which have been optimized in terms of the volume fraction of SiC, contact area between the components and presence of open porosity.
\end{abstract}

\section{Introduction}

Worldwide research in the field of thermal management materials has lead to development of high thermally conductive diamond-based composites. Among them, diamond-SiC have potentially a high thermal conductivity $(>400 \mathrm{~W} / \mathrm{m} / \mathrm{K})$, low density and, in contrast to the metal-based composites, CTE matched to the one of silicon [1].

These composites are produced in HTHP process [2], GPI or squeeze-casting [3], [4] and, more recently, by reactive microwave sintering [5]. The latter enables selective absorption of microwave energy by $\mathrm{Si}$, preventing graphitization of diamond. The as-grown $\mathrm{SiC}$ provides a mechanical stability of the composite and a path for heat transport between the diamonds. However, the composites obtained by microwave sintering of $\mathrm{Si}$ and diamond powders are characterized by a complex structure, Fig. 1, which needs to be optimized for their industrial applications.

The goal of this work is to develop a model which could be used to predict the effective thermal conductivity of diamond-SiC-Si porous and infiltrated composites with varying microstructures. To this end, a finite element method (FEM) has been used with representative unit cell shown in Fig.2. The FEM is a universal tool which can be applied to problems with various types of load, including mechanical, thermal stresses [6] or heat [7] and this method was used in the current work.

\section{Experimental}

A representative cell was proposed basing on the microstructures of the diamond-SiC-Si composites. The materials were synthesised by pressure-less microwave sintering of a mixture of diamond and silicon powders at $\mathrm{C} / \mathrm{Si}$ wt. ratio of 4/1. Open and closed porosity of the as-sintered composites of respectively 44 and 2 vol. \% was obtained using pycnometer and the Archimedes method. The porous composites were subsequently infiltrated with aluminium by squeeze-casting. The composites microstructures are shown in Fig. 1. For verification of the phase composition energy-dispersive X-ray analysis (EDXA/EDS) was used. The SiC crystals having a cube-like 
morphology occupy the diamonds faces next to remaining $\mathrm{Si}$, the amount of which depends on the extent of the reaction between silicon and carbon.

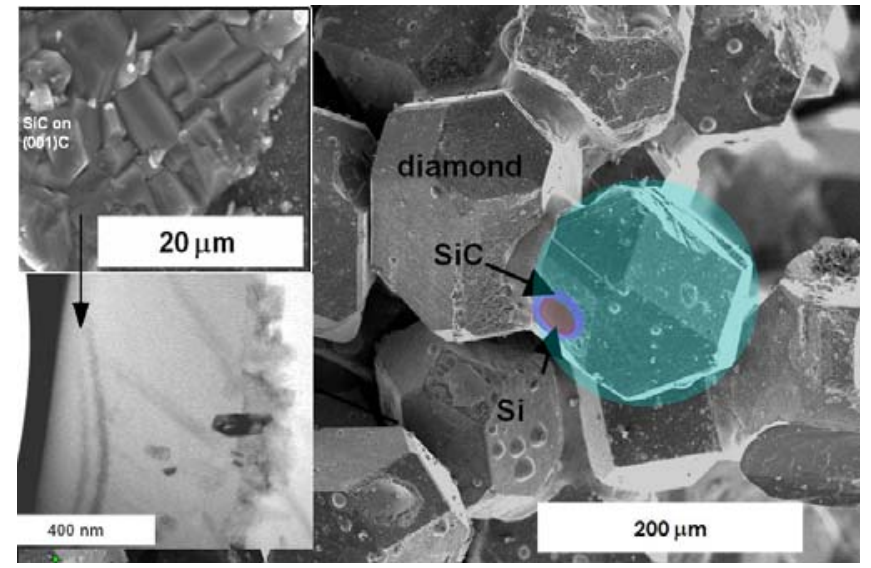

(a)

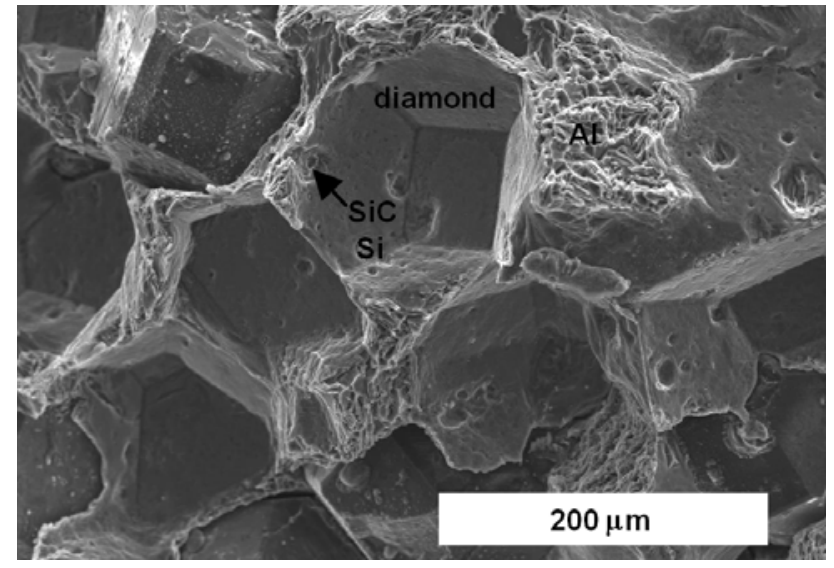

(b)

Fig. 1. SEM and TEM images of (a) porous and (b)Al-infiltrated diamond-SiC-Si composite

Thermal conductivity was modeled for a representative unit volume, RUV, of the composite in the form of a cubic cell with periodic boundaries. The diamond particles have been described as cut spheres linked by cylindrical bridges to six nearest neighbors. Fig. 2 shows $1 / 4$ of the cell where $a$ is the length of the side of the cube. This approach allows for flexibility in defining the dimensions of diamond, $\mathrm{SiC}$ and $\mathrm{Si}$, without changing the size of the RUV. The FEM modelling of the thermal properties of RUV was carried out using Ansys. The thermal analysis was based on the heat balance equation obtained from the rule of energy conservation. The $K_{\text {eff }}$ was obtained from the sum of heat fluxes through the voxels divided by the number of nods. Steady state load conditions were used to obtain the temperature distribution in the modelled volume. The temperature gradient applied between the upper and lower plane of the RUV was of $2{ }^{\circ} \mathrm{C}$. The following values of thermal conductivity were assumed in the computations: $K_{d}=2000, K_{S i C}=420, K_{S i}=130, K_{\text {por. }}=0, K_{A l}=$ $230(\mathrm{~W} / \mathrm{m} / \mathrm{K})$. The interfacial thermal resistance has been neglected for diamond-SiC interfaces.

The FEM model was used to study the apparent conductivity dependence on the following parameters: (1) relative amount of $\mathrm{SiC}$ and $\mathrm{Si}$ within the bridges linking diamonds $\left(V_{d}=\right.$ const), (2) volume fractions of the diamond, $\mathrm{SiC}, \mathrm{Si}$ and in the porosity and (3) infiltration of the pores with aluminium.

\section{Results and discussion}

The FEM mesh of the representative cell and the temperature distribution are shown in Fig. 2. The dimensions: $W, W_{S i C}$ and $W_{S i}$ correspond to the diameter of the diamond face, SiC disc and absolute diameter of $\mathrm{Si}$ ring, respectively, Fig. 3(a). The values of effective thermal conductivity as a function of the $\mathrm{SiC}$ bridge width, $W_{\text {sic }}$, the corresponding diamond/SiC interface area and relative volume fractions of the present phases are plotted in Fig. 4(a). Similarly, Fig. 4 (b) shows $K_{\text {eff }}$ as a function of the width, $W_{s i}$, and interface area between diamond and the hybrid SiC-Si bridge. A schematic representation of Al-infiltrated composites structure with and without the $\mathrm{SiC}$ bridges connecting the diamonds is given in Fig. 3(b).

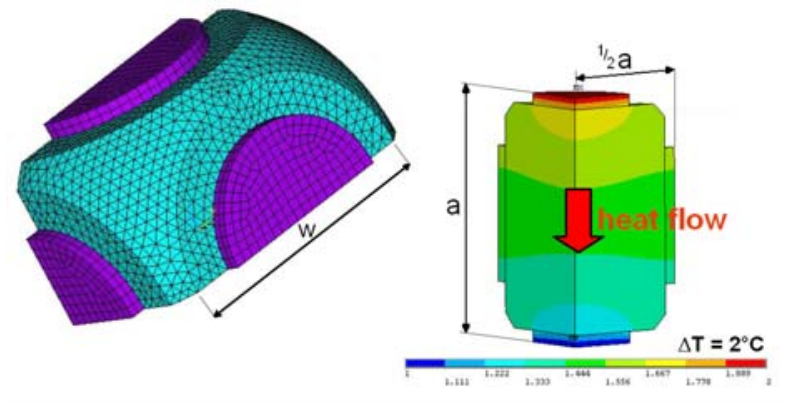

Fig. 2. FEM mesh and the temperature distribution across the representative unit volume. 


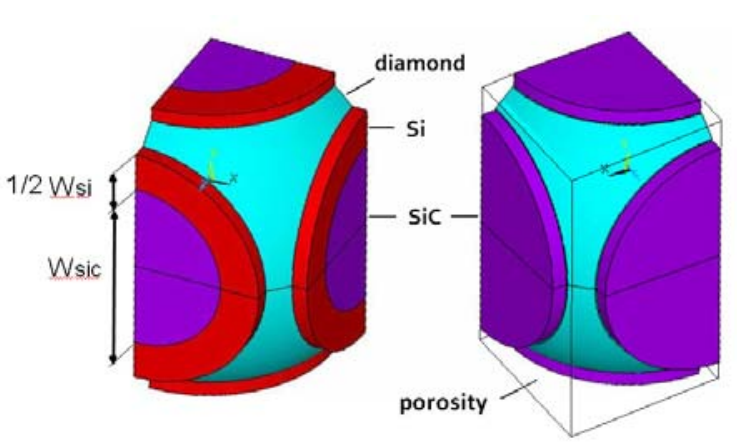

(a)

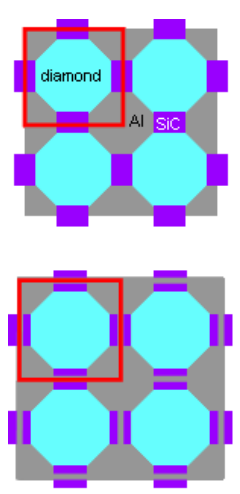

(b)

Fig. 3. (a) The representative cell with and without Si and (b) schematic illustration of the structure of diamondSiC-Al composite with SiC bridges linking the diamonds and with disconnected diamonds

It has been computed that the effective conductivity of the diamond-SiC composite, D-SiC, gradually increases with the $W_{S i C}$ and with the area of the SiC/diamond interface, $A_{S i C}$, Fig. 4(a). On the other hand, an increase of the volume fraction of Si substituting the $\mathrm{SiC}$ causes a decrease of the thermal conductivity in the Si-containing diamond-SiC composite, D-SiC-Si, Fig. 4(b). The low conductivity of silicon is limiting the effective conductivity of the composite. This implies that the content of Si must be minimized for heat sink applications.

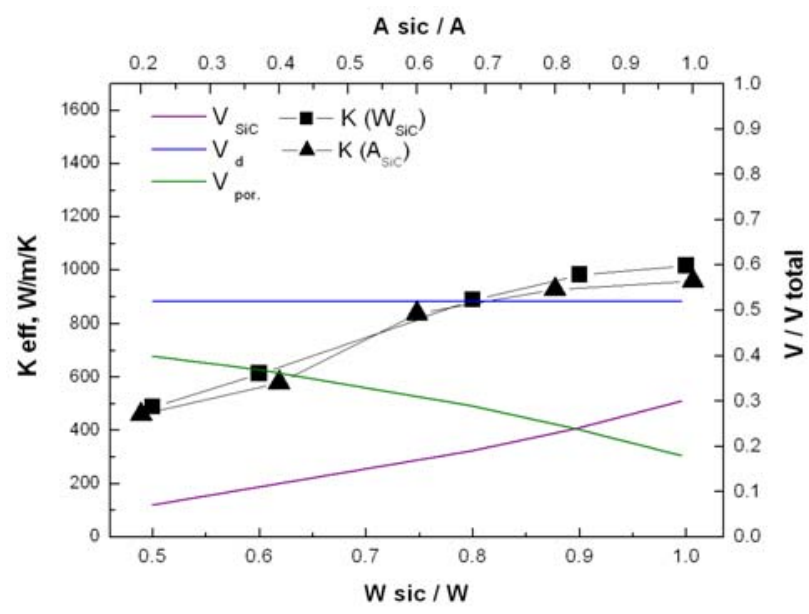

(a) diamond interface area, $A_{\mathrm{SiC}}$, and the volume of diamond, $V_{\mathrm{d}}$, silicon carbide, $V_{\mathrm{SiC}}$, silicon, $V_{\mathrm{Si}}$, and porosity, $V_{\text {por }}$ for: (a) diamond-SiC and (b) diamond-SiC-Si composite; $V_{\text {total }}=V_{\text {cell }}=a^{3}$

The $K_{\text {eff }}$ calculated for the infiltrated and porous composites with varying amount of Si in the $\mathrm{SiC}-\mathrm{Si}$ bridges are plotted in Fig. 5. One can see a decrease of $K_{\text {eff }}$ with the Si volume in both composites. In the case of SiC comprising 100 vol. \% of the bridge, the thermal conductivity of the porous diamond-SiC is similar to the conductivity of the infiltrated diamond-SiC-Al composite, Fig. 5.

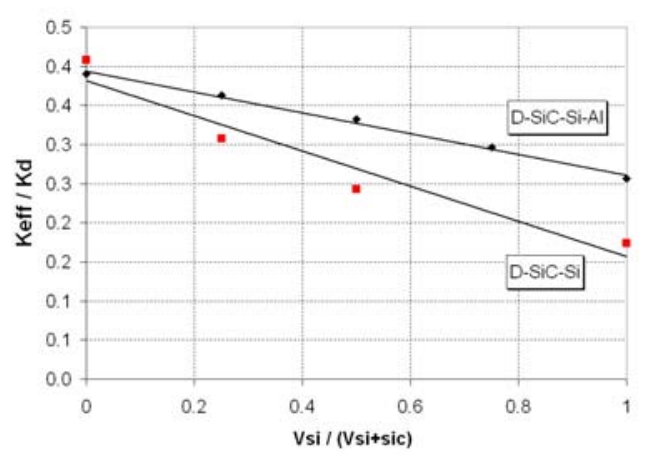

Fig. 5. Relative $K_{\text {eff }}$ for the non infiltrated and Al-infiltrated diamond-SiC-(Si) composites as a function of $\mathrm{Si}$ content 


\begin{tabular}{|c|c|c|c|}
\hline $\begin{array}{c}\mathrm{SiC} \\
\% \text { bridge }\end{array}$ & $\begin{array}{c}\mathrm{Si} \\
\% \text { bridge }\end{array}$ & $\begin{array}{c}\mathrm{Al} \\
\% \text { cell }\end{array}$ & $\begin{array}{c}K_{\text {eff }} \\
\mathrm{W} / \mathrm{m} / \mathrm{K}\end{array}$ \\
\hline 100 & - & 55, without $\mathrm{SiC}$ bridge & 510 \\
\hline 100 & - & 20, with SiC bridge & 870 \\
\hline- & 100 & non infiltrated & 600 \\
\hline 50 & 50 & non infiltrated & 950 \\
\hline
\end{tabular}

Table 1. The effective thermal conductivity of diamond-SiC-Si-(Al) composites for different RUV parameters

The results of the calculation reveal a strong contribution of high thermally conductive $\mathrm{SiC}$ to the total conductivity of the composite. The conductivity values obtained for various types of RUV are listed in Table 1. It can be noted that for disconnected diamond particles in aluminum matrix, Fig. 3(b), the conductivity is significantly lower than in the case where $\mathrm{SiC}$ is linking the diamonds. This rationalizes the design of a skeleton-like structure comprising diamonds connected by a wellconducting $\mathrm{SiC}$ rather than diamonds in a continuous $\mathrm{Al}$ matrix having a moderate thermal conductivity.

\section{Summary}

The FEM model allows for estimation of effective thermal conductivity of the diamond-SiC-(Si) composites as a function of the width of $\mathrm{SiC}$ bridges and interface area between diamond and SiC. The un-reacted silicon lowers the effective conductivity due to a three times lower thermal conductivity of $\mathrm{Si}(130 \mathrm{~W} / \mathrm{m} / \mathrm{K})$ with respect to $\mathrm{SiC}(420 \mathrm{~W} / \mathrm{m} / \mathrm{K})$. The $K_{\text {eff }}$ obtained for the composite with bridges built of $\mathrm{Si}$ approaches $500 \mathrm{~W} / \mathrm{m} / \mathrm{K}$. The same value of $K_{\text {eff }}$ is obtained for the composites with bridges built of $\mathrm{SiC}$ with two time smaller volume. Infiltration with $\mathrm{Al}$ does not contribute to the increase the $K_{\text {eff. }}$. The $\mathrm{SiC}$ on diamond works as an efficient heat path only when it percolates the entire structure. Finally, it can be pointed out that the results obtained here can be used to design new types of composites for industrial applications.

\section{Acknowledgements}

The work was supported by The Warsaw University of Technology, Faculty of Materials Science and Engineering and The International PhD School Switzerland-Poland.

\section{References}

[1] C. Zweben, Power Electonics Technology (2006)

[2] J. Qian, G. Vornin, T. W. Zerda, D. He, Y. Zhao, Journal of Materials Research 17 (2002)

[3] P. Ruch, O. Beffort, S. Kleiner, L. Webber, P. Uggowitzer, Composites Science and Technology 66 (2006)

[4] S. Kleiner, F. A. Khalid, P. Ruch, S. Meier and O. Beffort, Scripta Materialia 55 (2006)

[5] S. Leparoux, C. Diot, A. Dubach, S. Vaucher, Scripta Materialia 57 (2007)

[6] M. Iuga, F. Raether, J. European Ceramic Society 27 (2007)

[7] Y. Xu, K. Yagi, Computational Materials Science 30 (2004) 